\title{
Predictability and Accuracy between 3D Virtual Surgical Planning and Actual Outcomes in Orthognathic Surgery
}

\author{
Pampín Martínez MM, ${ }^{1 *}$ Guiñales Díaz de CevallosJ,, ${ }^{1}$ Del Castillo Pardo de Vera JL, ${ }^{1}$ Moreiras Sánchez AD, ${ }^{1,2}$ Dueso \\ Delgado V, ${ }^{1}$ CebriánCarreteroJL ${ }^{1,2}$
}

${ }^{1}$ Hospital Universitario La Paz, Madrid, Spain

${ }^{2}$ Hospital La Luz, Madrid, Spain

\begin{abstract}
Introduction: We are living a paradigm shift in orthognathic surgery. Several tools and technological advances are being implemented to help improve surgical experience and to offer better functional and aesthetical results. Virtual surgical planning is a revolutionary tool which helps us design the surgical plan preoperatively and establish a surgical protocol specifically designed for that patient. In this study, our goal is to validate the predictability of virtual surgical planning in orthognathic surgery, comparing the final results with the virtual plan.
\end{abstract}

Material and methods: We performed an ICP based super imposition of 3D models of the virtual planning preoperatively designed and the 3D model of the postoperative CBCT using free software (Slicer) in patients who underwent bimaxillary orthognathic surgery. The results of the super imposition were saved on the postoperative model and presented as a color-coded map. This was generated as a .vtk file that was exported to another free software that displayed the difference in $\mathrm{mm}$ in relevant cephalometric points (point A, point B, pogonion, left and right gonion and first upper molars) in the three axis ( $\mathrm{x}, \mathrm{y}$ and $\mathrm{z}$ ) between the postoperative result and the virtual plan. Then, we registered these results for all patients and analysed these data.

Results: A total of 41 patients were included. The median of the differences in mm between virtual planning and postoperative results were less than $1 \mathrm{~mm}$ for all cephalometric points, except for both gonion, where greater than $1 \mathrm{~mm}$ differences were found in the mediolateral (horizontal) direction. For the rest of landmarks, the highest differences were found at A point and pogonion in the anteroposterior direction $(0,83 \mathrm{~mm}$ and $0,78 \mathrm{~mm}$, respectively).

Conclusions: We found overall small and tolerable differences $(<1 \mathrm{~mm})$ between the planned movements and the postoperative results. The highest were found at the gonion in the mediolateral direction and mandibular rami, which could be explained by the torque of the proximal segment. The differences in A point and pogonion in the anteroposterior direction were the highest among the rest, which may be related to inaccuracy of the splint in this direction.

Keywords: Predictability, Orthognathic surgery, Virtual planning

\section{Introduction}

Success in orthognathic surgery depends not only on the technical aspects of the operation but to a larger extent on the formulation of a precise surgical plan, consistency and capability of achieving predictable, stable results. ${ }^{1} \mathrm{New} 3 \mathrm{D}$ tools have lately arisen to help us improve accuracy and results, such as virtual surgical planning, which has long been applied in craniofacial surgery but less so in maxillofacial surgery. ${ }^{2}$ Because of current improvement in imag ing and software, computer-assisted surgery has been increasingly used in orthognathic surgery to improve surgical outcomes. ${ }^{3}$

Traditionally, conventional planning of orthognathic surgery was conducted on the basis of a radiographic cephalometric analysis and mock surgery on plaster-cast dental models, mounted in a semi- adjustable articulator. ${ }^{4}$ This cast model surgery was complex, and difficulties may be encountered when correcting occlusal cant and facial asymmetries.
Quick Response Code:

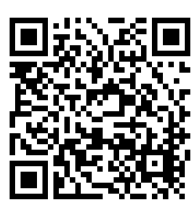

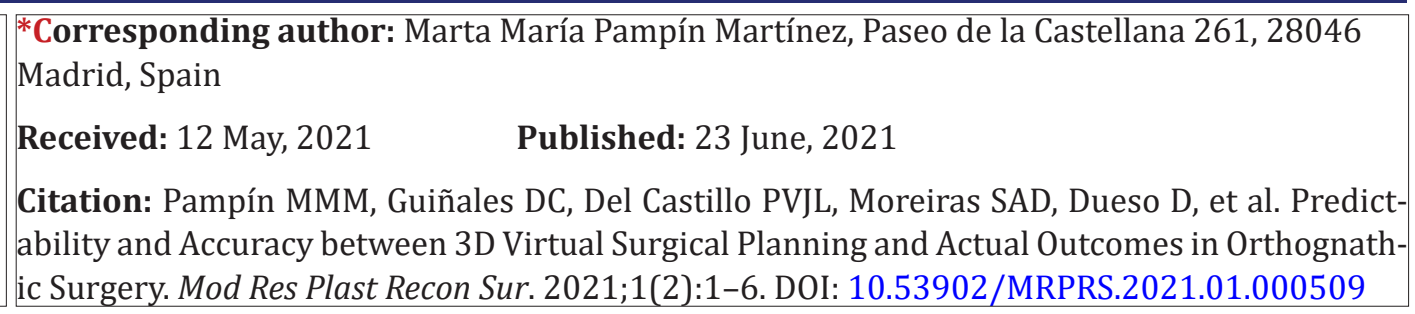


The development of computer-aided surgical simulation represents a paradigm shift in surgical planning for patients with cranio-maxillofacial deformities. ${ }^{5}$ Three-dimensional imaging-based planning systems enable the surgeon to establish necessary osteotomy planes preoperatively and assess different surgical scenarios ${ }^{1}$. With these, surgeons are able to design an accurate preoperative surgical plan that foresees limitations and inappropriate movements so that these are avoided in the operating room. There are many commercially available software programs for virtual surgical planning and simulation. ${ }^{6}$

However,the surgical planning may not necessarily reflect the actual surgical outcomes obtained and its accuracy and predictability must be established.

Our objective is to evaluate predictability in orthognathic surgery comparing the postoperative results to the virtual surgical plan preoperatively established in bimaxillary orthognathic patients and analyse these results. How predictable is the surgical plan we design virtually?

\section{Material and Methods}

We designed a retrospective study to analyse the predictability of virtual orthognathic comparing the postoperative results to the virtual planning. Inclusion criteria included patients who underwent bimaxillary orthognathic surgery at our department between October 2017 and December 2019; patients with available virtual planning models; patients where a Le Fort I osteotomy and bilateral sagittal split osteotomy were performed and patients with a postoperative CBCT within 1-month post-surgery. Patients with unavailable 3D models of the planning, without postoperative CBCT, patients who received a TMJ total prosthesis and patients who had another osteotomy distinct from a Le Fort I and bilateral sagittal split osteotomy were excluded. The main outcome variable was the difference in $\mathrm{mm}$ between the virtual plan and the actual surgical results obtainedin selected cephalometric points in the 3 axis: point A, point B, pogonion, both gonion and both upper right molars at the level of maxillary bone.

A total of 41 patients were included. No conflicts of interest are reported by the authors. Patients received a standard orthognathic workup including alginate impressions, facial analysis, preoperative CBCT in centric relation, facebow analysis and photographs. A virtual meeting was held by the surgeons to discuss the surgical plan with the prosthodontist. The surgical virtual planning was then designed using the Dolphin ${ }^{\circledR}$ software and surgical splints were designed and printed. Surgery was performed in a mandible-first sequence.The vertical position was controlled by the surgeon using a reference screw at the nasion. Osteosynthesis in the mandible was performed with a 4-screw plate and a bicortical screw, and 4 L-type plates on the maxilla. Postoperative antibiotics were administered, and a postoperative X-rays was performed to check for adequate condyle position.

First, we selected a group of relevant cephalometric points to calculate differences between planning and postoperative results by the mean linear distance difference, similarly to previous publi- cations. ${ }^{4,7}, 8$ These included: point $A$, point $B$, pogonion, both gonion and both upper right molars at the level of maxillary bone.

Then, the DICOM files of the postoperative CBCT were imported into the 3D Slicer software and a 3D model was generated in STL format, which was further refined and trimmed using the MeshMixer $®$ software for better similarity to the virtual planning STL. Then, both the planning STL and the postoperative STL model were loaded onto Slicer for superimposition.

First, a gross approximation was done using the "transforms" tool (Figure 1). Then, the "surface registration" tool was used for the superimposition of both models (Figure 2). The program uses an iterative closest point (ICP) algorithm, using the planning STL as reference.

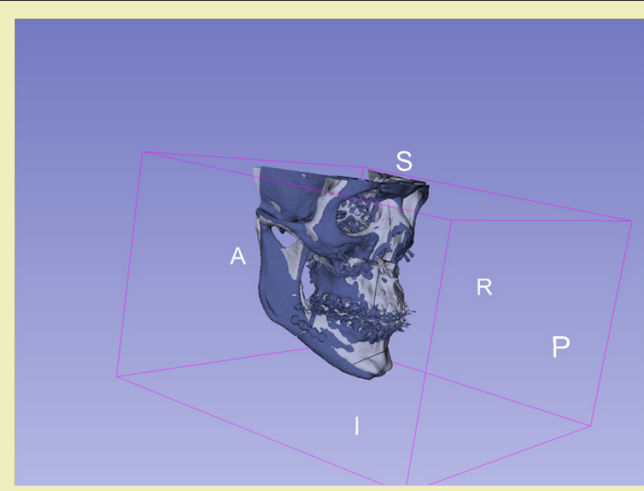

Figure 1: Gross approximation of both models using the transforms tool.

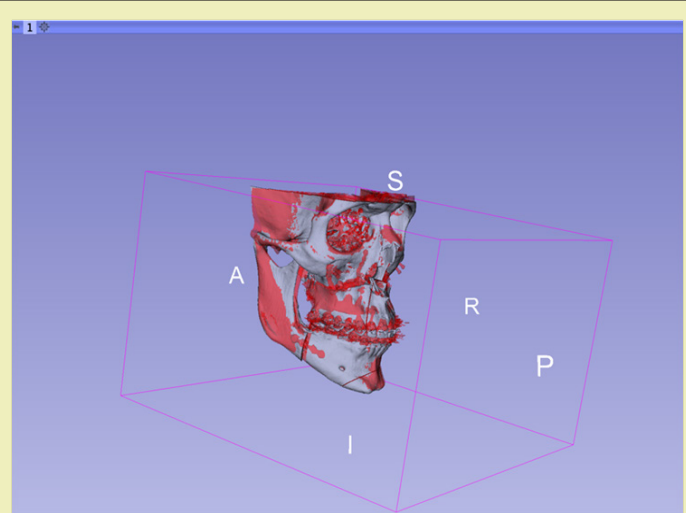

Figure 2: Surface registration of both models. The program uses an iterative closest point (ICP) algorithm, using the planning STL as reference.

Once the superimposition was completed, the "model to model distance" tool is used to calculate distances between both models and render the results on the postoperative model (Figure 3). Using the "Shape population viewer" extension in 3D Slicer, a color-coded map is displayed on the postoperative model showing the signed distances in different colours attending to the sign and magnitude of this value. This method assesses 3D changes of both surfaces (the virtual planning model and the postoperative model) by measuring the point-to-point distance of one mesh to the other and generating a color-coded distance map, similar to other authors. ${ }^{9}$ 


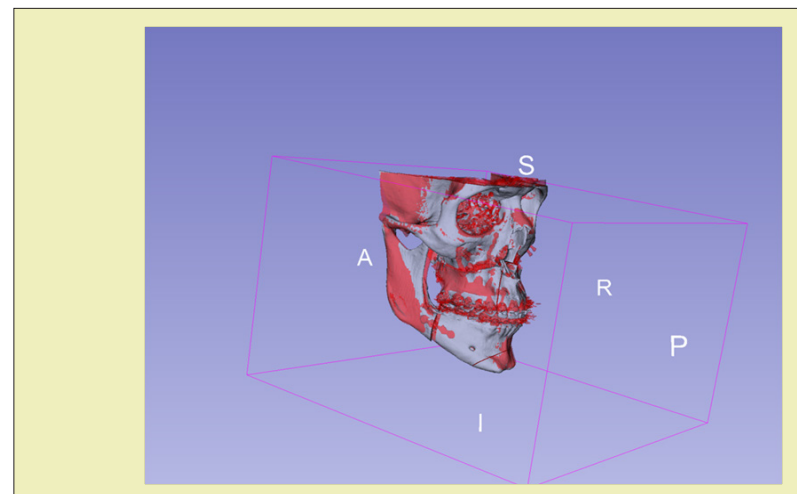

Figure 3: Model to Model distance calculates the distance between both models. We used the signed closes point mode to obtain signed distances.

In the color-coded distance maps, we designed a continuous colour-scale where blue was $-4 \mathrm{~mm}$, green $0 \mathrm{~mm}$ and red $+4 \mathrm{~mm}$. Positive colours (yellow-red) depict regions that are in front of the reference surface, which is the virtual planning model, indicating outward movements, and, negative colours (blue), indicate areas that are behind the reference surface, meaning backward movements

In Figure 4, an example of a colour map is presented, showing differences between both virtual planning and postoperative STLS. Each colour represents a value ranging from blue to red. If the cranial base appears green-coloured $(0 \mathrm{~mm})$ the quality of the superimposition is good.

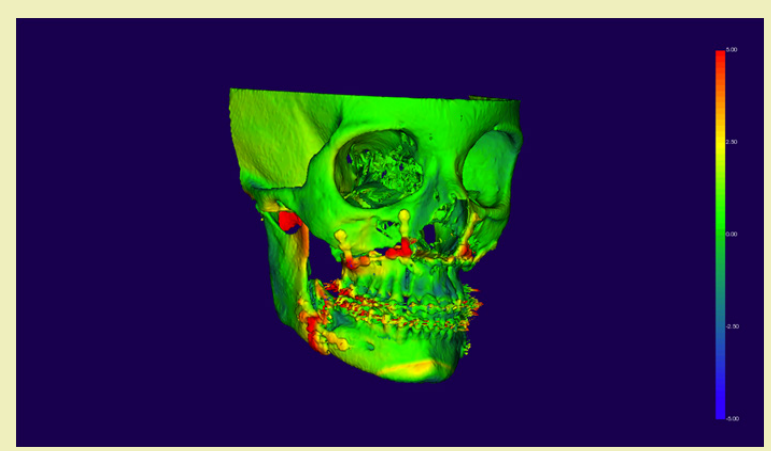

Figure 4: Color-coded maps of the distances in another model. A continuous color-coded scale is designed where blue indicates negative distance differences and red positive distance differences.

Then, the .vtk file of the postoperative model, which contains the information of the distances between both models, is exported to the open-source Paraview application. We used the "Hover points on" tool to display information of thedistance difference in the cephalometric points selected in the three axis " $x$ " (mediolateral), "y" (anteroposterior) and "z" (infero superior) (Figure 5). The cephalometric points selected were analysed by placing the mouse over the point on the 3D model and the information regarding the distance in the three axis for that point was displayed. This measurement was done 3 times by two different surgeons and the mean value of these measurements was selected.

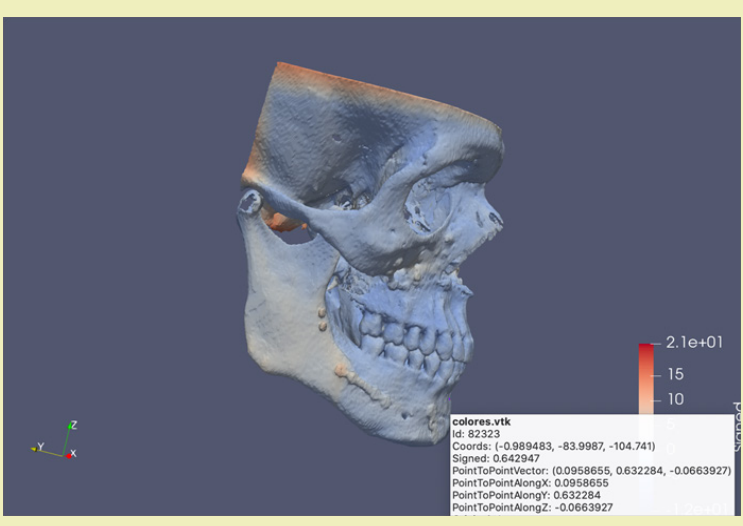

Figure 5: Information of the differences is displayed on the postoperative model.

For the statistical analysis we first analysed if our population followed a normal distribution. For this, we performed the Kolmogorov-Smirnov test. A non-normal distribution was followed for most data, so non-parametric tests were decided. We registered the distance difference for all patients in points A, B,pogonion, both gonion and both upper molars and then we calculated the median and percentiles of this value for all cephalometric points.

\section{Results}

A total of 41 patients were selected that satisfied the inclusion criteria. Mean age was 29 years, ranging from 17 to 55 years. 11 men and 30 women were included.

Table 1 shows the median and the 25 and 75 percentiles of the differences between virtual planning and postoperative results for each cephalometric point in the three axis for all patients. These results are also displayed on Figure 6. The x axis represents the mediolateral direction, the $y$ axis the anteroposterior direction and the $\mathrm{z}$ axis the inferosuperior or vertical direction.

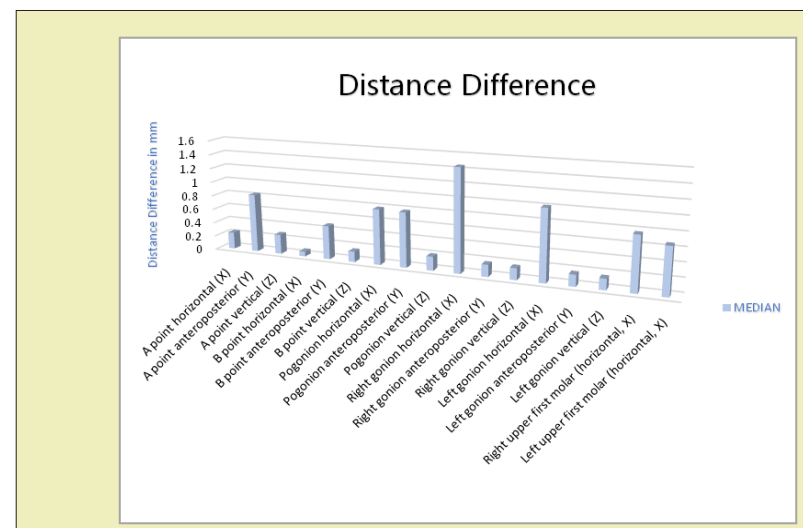

Figure 6: Graphic displaying information about median distance difference for all cephalometric points.

The overall analysis showed good accuracy, being all median differences less than $1 \mathrm{~mm}$, except for both left and right gonion in the mediolateral direction, which were the highest: 1,01 and 1,45, respectively. This suggests postoperative flaring of the mandibular angle, which was confirmed in the color-coded maps where this region appeared in yellow-red for these patients. 
Table 1: Median and 25 and 75 percentiles for all differences between surgical planning and postoperative results.

\begin{tabular}{|c|c|c|c|}
\hline $\mathrm{N}=41$ patients & Percentile 25 & MEDIAN & Percentile 75 \\
\hline A point horizontal $(X)$ & 0,077 & 0,239 & 0,360 \\
\hline A point anteroposterior $(Y)$ & 0,297 & 0,835 & 1,400 \\
\hline A point vertical (Z) & 0,075 & 0,280 & 0,600 \\
\hline B point horizontal $(X)$ & 0,020 & 0,070 & 0,330 \\
\hline$B$ point anteroposterior $(Y)$ & 0,162 & 0,480 & 0,885 \\
\hline B point vertical $(Z)$ & 0,365 & 0,150 & 0,292 \\
\hline Pogonion horizontal $(X)$ & 0,13 & 0,79 & 0,19 \\
\hline Pogonion anteroposterior $(Y)$ & 0,175 & 0,780 & 1,160 \\
\hline Pogonion vertical (Z) & 0,070 & 0,200 & 0,625 \\
\hline Right gonion horizontal $(X)$ & 0,365 & 1,455 & 2,700 \\
\hline Right gonion anteroposterior $(Y)$ & 0,030 & 0,170 & 0,487 \\
\hline Right gonion vertical (Z) & 0,038 & 0,165 & 0,690 \\
\hline Left gonion horizontal $(X)$ & 0,415 & 1,010 & 1,439 \\
\hline Left gonion anteroposterior $(Y)$ & 0,075 & 0,165 & 0,485 \\
\hline Left gonion vertical (Z) & 0,039 & 0,155 & 0,602 \\
\hline Right upper first molar (horizontal, $X$ ) & 0,257 & 0,770 & 1,445 \\
\hline Left upper first molar (horizontal, X) & 0,186 & 0,665 & 1,222 \\
\hline
\end{tabular}

For the rest of cephalometric points, the highest mean differences were seen at A point and pogonion in the anteroposterior direction, which were 0,83 and 0,78 , respectively. Overall accuracy in the vertical direction was good for all cephalometric points. Also, differences at the bone over the upper first molars were higher: 0,77 and 0,66 for the right and left molars. Differences at this level might be influenced by dentoalveolar movements during the postoperative orthodontic treatment.

\section{Discussion}

Conventional planning of orthognathic surgery was carried out on the basis of an X-ray cephalometric analysis and mock surgery on plaster cast dental models mounted in a semi-adjustable articulator. ${ }^{4}$ However, this method is complex and arduous and has a high risk of error that may lead to suboptimal results. In comparison, virtual surgical planning enables precise analysis of a 3D model that represents the clinical situation and facilitates diagnosis and treatment planning. ${ }^{10,11}$ Since Swennen ${ }^{11}$ initiated the 3D cephalometric analysis and treatment planning, 3D virtual planning is replacing the 2D cephalometric analysis.

With 3D virtual surgical planning surgeons cananalysewhich movements are the best to achieve the desired occlusion and aesthetics using a computer, assessing different scenarios and the advantages and limitations of each. This is particularly helpful for patients with significant facial deformity and asymmetry. ${ }^{3}$ Using 3D models, all of the procedures for diagnosis and surgical splint production can be simulated, intermediate assessment can be conducted, and the intermediate and final surgical splints can be produced. Therefore, errors encountered during laboratory procedures are minimal. ${ }^{12}$

Nevertheless, differences still exist between the virtual planning and surgical execution due to the complexity of movements and transfer of data throughout splints from the virtual planning to the surgery. As any tool, the reliability and predictability of virtual surgical planning must be tested.

In the literature, many studies have tried to evaluate the accuracy of virtualplanning in orthognathic surgeryusing different protocols. These protocols and methodologies vary from linear/angular measurements, surface to surface differences or virtual triangles. The most commonly used approach is linear and angular measurements, which relies on accurately identifying cephalometric landmarks, which is prone to human error especially when if it has to be done on the preoperative and postoperative object. ${ }^{13}$ Color-coded distances maps are a visual analytical tool that displays the distance between two 3D surface meshes and is generally included in most software.

Ho and colleagues ${ }^{14}$ analysed the accuracy of virtual surgical planning by calculating the root-mean square difference (RMSD) and found that the results were acceptable, with RMSD $0.63 \pm 0.25$ $\mathrm{mm}$ for the maxilla and $0.85 \pm 0.41 \mathrm{~mm}$ for the mandible.

Tucker, et al. ${ }^{15}$ evaluated the accuracy of virtual planning based on the surface distance differences between the plan and the actual outcome on 11 different regions of the maxilla and mandible. The method was done using a surface-to-surface best fit of the two virtual models aligning the base of the skull and measuring the distance between the planned and actual outcome post-operatively. They found no statistically significant difference between the simulated and the actual surgical models in all 11 regions of interest, being less than $0.5 \mathrm{~mm}$ except for the left lateral maxilla $(0.536 \mathrm{~mm})$.

Hsu, et al. ${ }^{7}$ in 2013 studied differences in the position and orientation between the planned and postoperative outcomes in 65 patients in 3 different 3 centres who underwent bimaxillary orthognathic surgery using a CASS protocol. They found that the largest 
positional difference in the maxilla was $1.0 \mathrm{~mm}$ and $1.1 \mathrm{~mm}$ in the mandible, showing overall excellent positional and orientation accuracy for the maxilla and mandible.

Stokbro, et al. ${ }^{4}$ studied 30 patients who had undergone bimaxillary orthognathic surgery, with and without segmentation and genioplasty, and found all mean linear differences for the maxilla, mandible and chin segment to be within $0.5 \mathrm{~cm}$. They also found that the mean precision, measured as the standard deviation, was smallest superoinferiorly, followed by mediolateral deviation and finally anteroposteriorly. Precision was also most accurate in the mandible, slightly less in the maxilla and least in the chin segment, probably due to the mandible-first sequence.

Cevidanes, et al. ${ }^{16}$ and Hajeer, et al. ${ }^{17}$ quantified 3D displacement using the $\mathrm{x}, \mathrm{y}$, $\mathrm{z}$ vectors of landmark displacement, similarly to our study. Kawamata, et al. ${ }^{18}$ described methods referring to both linear and angular measures. However, these measures do not reflect what happens along the whole surface.16 For this reason, color-coded maps are needed to display information on what is happening along the whole surface model.

De Riu, et al. ${ }^{6}$ performed a retrospective study in 49 patients undergoing bimaxillary orthognathic surgery and performed a cephalometric analysis. Average error detected was $1.98 \mathrm{~mm}$ for linear measures and $1.19^{\circ}$ for angular measures. They found significant differences between planned and achieved anterior facial height ( $\mathrm{p}$ $=0.033$ ) thatcould be compensated for with genioplasty. The authors concluded that the problem was caused by the virtual model of the soft tissues, which made it difficult to manage the vertical dimension. They also reported differences in SNA and SNB between planned and actual measurements were found to besignificant as well. This may be related to imperfect condylar seating, causing unintentional changes to the surgical plan and under-advancement of the jaws.

Lee and colleagues ${ }^{19}$ and Stockbro ${ }^{4}$ also analysed the importance of proper condylar position and suggested that the position might be alteredduring surgeryby muscle tone and gravity as the patient is placed in the supine position, which affects correct condylar seating.

In their systematic review, Ali Alkhayer, et al. ${ }^{20}$ analysed 12 papers regarding accuracy analysis of virtual surgical planning. The accuracy values for pitch, yaw, and roll $\left({ }^{\circ}\right)$ were $(<2.75,<1.7$ and $<1.1)$ for the maxilla, respectively, and $(<2.75,<1.8,<1.1)$ for the mandible. They observed that calculation of the linear and angular differences between the virtual plan and postoperative outcomes was the most frequented method used for accuracy assessment and a difference less than $2 \mathrm{~mm} /{ }^{\circ}$ was considered acceptable and accurate. They concluded that virtual planning appears to be more accurate, especially in terms of frontal symmetry.

The method we applied in this study can express more 3D shape information in comparison to those that were based solely on the calculations of linear and angular distances, offering valuable information in the three axis for the cephalometric points selected and visual data in the color-coded maps. We found overall good accuracy, being all median of thedistance differences below $1 \mathrm{~mm}$, except for the "x" axis (mediolateral direction) at both gonion.The greatest values for the rest of the landmarks were found in the "y" axis (anteroposterior) at Apoint and pogonion. In the literature, a difference of less than $2 \mathrm{~mm}$ between the virtual surgical planning and the actual postoperative results has been considered clinically acceptable by many authors. ${ }^{4,15}$ However, we believe that this statement must be taken cautiously, since it highly depends on the quantity of movement; it's not as clinically irrelevant for an advancement of $4 \mathrm{~mm}$ than $8 \mathrm{~mm}$.

We noticed that flaring of the gonion occurred, with differences in the mediolateral direction for both right and left gonion beinggreater than $1 \mathrm{~mm}$. This was confirmed in the color-coded maps, where the rami and gonial regions appeared in yellow-red in several patients. This might suggest that some quantity of flaring occurs after the sagittal split osteotomy of the mandible, producing torque of the proximal segment, which may alter the results, mostly in the mediolateral and anteroposterior directions. As stated by many authors, inadequate condylar seating can significantly influence mandibular positioning and remains a source of inaccuracyin orthognathic surgery, so special attention should be paid to condylar position. We believe this needs further studies to confirm this hypothesis.

Regarding the limitations of this study, it is important to take into account that it is a retrospective study and that, as stated by many authors, the selected landmarks for analysis need to be identified multiple times on the models, which is prone to human error. Another source of limitation may be correlated to erroneous data on the surface mesh (for example, streak artifacts or surface roughness) which would have a marked effect on the measurements. ${ }^{9}$

As improvements to this study, measurements in more cephalometric and dental landmarks could be done to improve the analysis. Different assessment methods could also be used to overcome observer-dependent landmark identification errors. Finally, we believe it would be interesting to direct further studies to analyse what happens in the proximal segment and, furthermore, in the condyle.

\section{Conclusion}

The differences between the surgical simulation and the actual postoperative results were less than $1 \mathrm{~mm}$ for most cephalometric landmarks, indicating overall acceptable predictability of the virtual surgery. Higher differences were found at the anteroposterior direction at A point and pogonion, which could be related of inaccuracy of the splint used in the surgery. Nonetheless, the values were still lower than $1 \mathrm{~mm}$, which is very acceptable.

However, we highlight the importance of paying attention to the proximal segment and gonial flaring when manipulating the mandible, where the highest differences (greater than $1 \mathrm{~mm}$ ) were found. We believe this could be explained by torque of the proximal segment and further studies are required to confirm this hypothesis and analyse what happens with the proximal segment in the longterm and its relation to postoperative relapse. 
In conclusion, we believe that virtual planning is a weapon of doubtless utility and importance that makes orthognathic surgery a more straightforward procedure, increasing its predictability and precision to obtain better functional and aesthetical results in our patients.

\section{Acknowledgments}

None.

\section{Funding}

This research did not receive any specific grant from funding agencies in the public, commercial, or not-for-profit sectors.

\section{Conflicts of Interest}

Author declares that there is no conflict of interest.

\section{References}

1. Quereshy FA, Baur DA, Levintov N, et al. Role of Virtual Surgical Planning in Increased Predictability of Orthognathic Surgery. J Den Max Surg. 2015;2(1):136-141.

2. Lo LJ, Marsh JL, Vannier MW, et al. Craniofacial computer-assisted surgical planning and simulation. Clin Plast Surg. 1994;21(4):501-516.

3. Chang HW, Lin HH, Chortrakarnkij $\mathrm{P}$, et al. Intraoperative navigation for single-splint two-jaw orthognathic surgery: From model to actual surgery. J Craniomaxillofac Surg. 2015;43(7):1119-1126.

4. Stokbro K, Aagaard E, Torkov 0, et al. Surgical accurcy of threedimensional virtual planning: a pilot study of bimaxillary orthognathic procedures including maxillary segmentation. Int J Oral Maxillofac. Surg. 2016;45(1):8-18.

5. Zinser MJ, Mischkowski RA, Dreiseidler T, et al. Computer-assisted orthognathic surgery: waferless maxillary positioning, versatility, and accuracy of an image-guided visualisation display. Br J Oral Maxillofac Surg. 2013;51:827-833.

6. De Riu G, Virdis PI, Meloni SM, et al. Accuracy of computer- assisted orthognathic surgery. J Craniomaxillofac Surg. 2017;46:293-298.

7. Hsu SS, Gateno J, Bell RB, et al. Accuracy of the computer-aided surgical simulation (CASS) system in the treatment of patients with complex craniomaxillofacial deformity: a pilot study. J Oral Maxillofac Surg. 2007;65:248-254.
8. Jabar N, Robinson W, Goto TK, Kambay BS. The validity of using surface meshes for evaluation of three-dimensional maxillary surgi- cal changes. Int J Oral Maxillofac Surg. 2015;44:914-920.

9. Baker SB, Goldstein JA, Seruya M. Outcomes in computer-assisted surgical simulation for orthognathic surgery. J Craniofac Surg. 2012;23:509-513.

10. Swennen GR, Mollemans W, Schutyser F. Three-dimensional treatment planning of orthognathic surgery in the era of virtual imaging. J Oral Maxillofac Surg. 2009;67:2080-2092.

11. Tran NH, Tantidhnazet S, Raocharernpron S, et al. Accuracy of ThreeDimensional Planning in Surgery-First Orthognathic Surgery: Planning Versus Outcome. J Clin Med Res. 2018;10(5):429-436.

12. Makram M, Kamel H. Reeb graph for automatic 3D cephalometry. IJIP. 2014;8(2):17-29.

13. Ho CT, Lin HH, Liou EJ, et al. Three-dimensional surgical simulation improves the planning for correction of facial prognathism and asymmetry: a qualitative and quantitative study. Sci Rep. 2017;7: p.40423.

14. Tucker S, Cevidanes LH, Styner M, et al. Comparison of actual surgical outcomes and 3-dimensional surgical simulations. J Oral Maxillofac Surg. 2010;68:2412-2421.

15. Cevidanes LHS, Bailey J, Tucker GR, et al. Superimposition of 3D conebeam CT models of orthognathic surgery patients. Dentomaxillofacial Radiology 2005;34:369-375.

16. Hajeer MJ, Ayoub AF, Millett DT, et al. Three-dimensional imaging in orthognathic surgery: The clinical application of a new method. Int J Adult Orthod Orthognath Surg. 2002;17:318- 330.

17. Kawamata A, Fujishita M, Kuniteru N, et al. Three-dimensional computed tomography evaluation of postsurgical condylar displacement after mandibular osteotomy. Oral Surg Oral Med Oral Pathol Oral Radiol Endod. 1998;85:371-376.

18. Lee CY, Jang CS, Kim JW, et al. Condylar repositioning using centric relation bite in bimaxillary surgery. Korean J Orthod. 2013;43:74-82.

19. Alkhayer A, Piffko J, Lippold C, et al. Accuracy of virtual planning in orthognathic surgery: a systematic review. Head \& Face Medicine. 2020;16:34.

20. Donatsky O, Bjorn-Jorgensen J, Holmqvist-Larsen M, et al. Computerized cephalometric evaluation of orthognathic surgical precision and stability in relation to maxillary superior repositioning combined with mandibular advancement or setback. J Oral Maxillofac Surg. 1997:55:1071-1079. 